\title{
Pre-operative direct and inverse clinical characteristic variations by age do not resolve up to 24 months following open roux-en-y gastric bypass
}

\begin{abstract}
Background: Today open Roux-en-Y gastric bypass (ORYGB) is reserved for abdomens too complex or obesity too severe for laparoscopic surgery. However, outcomes by age in ORYGB are unknown.

Objective: Identify variation by age in ORYGB patients.

Setting: Independent database.

Methods: 5389 ORYGB BOLD patients was analyzed retrospectively by age: $<30$ (591), 30-40 (1252), 40-50 (1527), 50-60 (1388), 60-70 (592) and >70 (39). Statistics: ANOVA/ general linear model.

Results: Baseline female/male $(\mathrm{p}<0.01)$, weight, BMI, African-American, Hispanic, Medicaid, Private insurance, self-pay $(\mathrm{p}<0.0001)$, PCOS, PTC $(\mathrm{n}=10)$ varied inversely with age. Medicare, CHF, HTN, angina, LEE, PVD, IHD, dyslipidemia, OHS, diabetes, gout, IFS, and unemployment varied directly. Caucasian, Asian, Other race, DVT/PE, hernia, cholelithiasis, GERD, panniculitis, liver disease, SUI, MSP, fibromyalgia, alcohol and tobacco abuse, depression, PI, MHD ( $\mathrm{n}=17)$ vary non-linearly. At 12 months, in spite of equal BMI by age, CHF, HTN, angina, PVD, dyslipidemia, cholelithiasis, SUI, diabetes, gout, IFS $(n=10)$ varied directly. PCOS and PTC varied inversely, and LEE, OSA, hernia, MSP, tobacco abuse, depression, MHD varied non-linearly. 24 months CHF, HTN, angina, dyslipidemia, diabetes, IFS, and MSP vary directly. PTC and tobacco abuse varied inversely. LEE, OSA, PHTN, hernia, PCOS, and tobacco abuse varied non-linearly.

Conclusions: ORYGB patients vary dramatically by decade of age. Pre-operatively 10 comorbidities varied inversely, 13 directly, and 17 non-linearly by age. In spite of equal BMI by age at 24 months, important co-morbidities CHF, HTN, angina, dyslipidemia, diabetes varied directly, tobacco inversely, and OSA non-linearly. This advance clinical knowledge of age variation can aid ORYGB management.
\end{abstract}

Keywords: open roux-en Y gastric bypass, ORYGB, obesity, age variations
Volume II Issue 5 - 202I

\author{
Ajay Menon, David Kelter, Gus J Slotman \\ Department of Surgery, Inspira Health Network, USA \\ Correspondence: Gus J Slotman, Director of Clinical \\ Research, Clinical Professor of Surgery RowanSOM \\ Inspira Health Network, 1505 West Sherman Avenue, Suite B \\ Vineland, NJ 08360, USA, Tel 856-64I-8635, \\ Fax 856-64I-8636, Email slotmang@ihn.org
}

Received: September 18, 202 | | Published: October 04, 202 |
Abbreviations: BMI, body mass index; AA, African-American; CHF, congestive heart failure; HTN, hypertension; LEE, lower extremity edema; PVD, peripheral vascular disease; IHD, ischemic heart disease; DVT/PE, deep vein thrombosis/pulmonary embolism; OHS, obesity hypoventilation syndrome; OSA, obstructive sleep apnea; IFS, impaired functional status; PCOS, polycystic ovarian syndrome; PTC, pseudotumor cerebri; GERD, gastroesophageal reflux disease; SUI, stress urinary incontinence; MSP, musculoskeletal pain; PI, psychological impairment; MHD, mental health diagnosis

\section{Introduction}

In today's bariatric surgery practice, Open Roux-en-Y gastric bypass (ORYGB) typically is reserved for medically or surgically complex patients who are deemed unfit for the usual laparoscopic approach. ${ }^{1}$ The most reliable predictors of a patient undergoing ORYGB are steatohepatitis, large waist size, BMI, preoperative weight, diabetes mellitus, and massive intraabdominal adhesions from various etiologies including prior operations. ${ }^{2}$

Functional decline of metabolic homeostatic processes over time is a major characteristic of the aging process. Aging and associated increasing visceral adiposity are etiological risk factors contributing to the development of insulin resistance, metabolic syndrome, type 2 diabetes mellitus, cardiovascular disease, and stroke. ${ }^{2}$ Highlighting this concept, metabolic syndrome increased from $6.7 \%$ in patients aged 20 through 29 to $43.5 \%$ for patients aged 60 to $69 .{ }^{3}$ Considering the elderly are the fastest growing segment of the population, with those age 60 or greater estimated to compose nearly 2 billion people globally by the year 2050 , and the exacerbated effects of obesity the oldest cohorts, the interaction of age with bariatric surgery may be increasingly consequential. ${ }^{4}$

In the subset of patients undergoing surgical management of their obesity, frequency of resolution of obesity-related comorbidities is associated with sex, insurance type, and race. Following laparoscopic Roux en Y gastric bypass (LRYGB) women retained abdominal and mental health weight-related problems more than men, but men drank more and resolved liver disease, important cardiopulmonary and vascular issues, metabolic derangements (diabetes, gout, dyslipidemia), and functional impairment significantly less than women. ${ }^{5}$ Davis et al discovered that private health insurance and self-pay patients benefitted more from ORYGB than did Medicare 
or Medicaid insured. ${ }^{6}$ Wood and co-investigators reported higher complication rates among African-Americans undergoing bariatric surgery, but comorbidity remission was similar to that of casematched cohorts. ${ }^{7}$ Regarding age and bariatric surgery, Hammond et al analyzed outcomes following LRYGB in septuagenarians, finding mixed results in this older population, limited to hypertension, hyperlipidemia, and diabetes. ${ }^{8}$

Whether or not clinical presentation and post-operative resolution of weight-related problems vary by age in the medically and surgically complex subset of patients who undergo ORYGB is unknown. The objective of this study was to identify age variation in relation to pre-operative clinical characteristics and resolution of comorbidities following surgery in patients necessitating ORYGB.

\section{Methods}

With the approval of the Data Access Committee of the Surgical Review Corporation and the IRB of Our Lady of Lourdes Medical Center, Camden NJ, pre-operative data and post-operative follow-up information through 24 months from the Surgical Review Corporation's Bariatric Outcomes Longitudinal Database (BOLD) on 5389 patients who underwent ORYGB from June 1, 2007 to December 31, 2010 was analyzed retrospectively by age: $<30(\mathrm{n}=591), 30-40(\mathrm{n}=1252)$, 40-50 $(n=1527), 50-60 \quad(n=1388), 60-70 \quad(n=592)$ and $>70 \quad(n=39) .{ }^{9}$ Data included age, weight, BMI, and 33 weight-related medical conditions: deep vein thrombosis/pulmonary embolism (DVT/ $\mathrm{PE})$, congestive heart failure (CHF), hypertension (HTN), angina, lower extremity edema (LEE), peripheral vascular disease (PVD), dyslipidemia, obstructive sleep apnea (OSA), obesity hypoventilation syndrome (OHS), asthma, pulmonary hypertension (PHTN), ischemic heart disease (IHD), abdominal hernia, cholelithiasis, gastroesophageal reflux disease (GERD), abdominal panniculitis, liver disease, stress urinary incontinence (SUI), diabetes mellitus, gout, polycystic ovarian syndrome (PCOS), pseudotumor cerebri

Table I Pre-operative variation of sex, race, \& insurance data (\%) by age
(PTC), back pain, impaired functional status (IFS), musculoskeletal pain (MSP), fibromyalgia, alcohol abuse, tobacco abuse, substance abuse, depression, psychological impairment (PI), mental health diagnoses (MHD), unemployment, and support group.

\section{Statistical analysis}

The differences in the age groups were examined at each time point using t-test for continuous variables (weight and BMI) and chi-square test to examine association of age decade with categorical variables (co-morbidities). To find the outcomes differences in the age groups for continuous values while adjusting for baseline value, a linear model was created with sex and baseline value as factors, for each post-baseline time point. Pair-wise comparisons were examined using least squares means (baseline adjusted means) calculated from the model to find differences in the age groups while adjusting for baseline. The differences in obesity co-morbidities with age were examined by using a categorical model while adjusting for baseline co-morbidities in a similar fashion but using a more appropriate categorical model. ${ }^{10}$ Statistically significant differences between the age groups were defined as varying directly with age when the variable increased linearly from $<30$ years to $60-70 />70$ years. Inverse variations were defined with the variable highest among $<30$ years patients and lowest in 60-70/>70 years. Non-linear variation was defined as statistically significant differences by age that were highest or lowest in 40-60 years.

\section{Results}

Demographics of patient age, sex, race, and insurance data are listed in Table 1. Medicaid, Private, and Self-Pay insurance all vary inversely to age $(\mathrm{p}<0.0001)$. Medicare insurance was highest in the $>70$ age group $(54.55 \%)$. Private insurance was highest in the $<30$ age group $(83.11 \%)$. Race varied non-linearly by age for Caucasian, Asians, and Other; and inversely by age for African-American (12.01\% in $<30$ age group) and Hispanics ( $15.06 \%$ in $<30$ age group)

\begin{tabular}{|c|c|c|c|c|c|c|c|c|c|c|c|}
\hline Age Group (n) & Female & Male & AA & Caucasian & Other & Asian & Hispanic & Medicaid & Medicare & Private & Self-Pay \\
\hline$<30(591)$ & 81.39 & $|8.6|$ & 12.01 & 59.39 & 13.37 & 0.17 & 15.06 & 10.53 & 3.07 & 83.11 & 3.29 \\
\hline $30-40$ (1252) & 77.56 & 22.44 & 14.06 & 60.86 & 11.26 & 0 & 13.82 & 10.43 & 5.41 & 82.13 & 2.03 \\
\hline $40-50$ (1527) & 74.26 & 25.74 & 9.17 & 71.05 & 11.13 & 0.07 & 8.58 & 9.88 & 9.71 & 79.35 & 1.06 \\
\hline $50-60(1388)$ & 75.5 & 24.5 & 6.77 & 77.95 & 10.95 & 0.14 & 4.18 & 6.65 & 11.91 & 79.78 & 1.66 \\
\hline $60-70$ (592) & 72.47 & 27.53 & 3.72 & 78.72 & 15.88 & 0.17 & 1.52 & 5.45 & 26.49 & 67.57 & 0.5 \\
\hline$>70$ (39) & 76.92 & 23.08 & 0 & 58.97 & 41.03 & 0 & 0 & 0 & 54.55 & 45.45 & 0 \\
\hline$p$-Value & 0.0032 & 0.0032 & $<.0001$ & $<.0001$ & $<.0001$ & $<.0001$ & $<.0001$ & $<.0001$ & $<.0001$ & $<.0001$ & $<.0001$ \\
\hline
\end{tabular}

Weight and BMI by age in ORYGB patients are depicted in Table 2. Pre-operatively these variables varied inversely with age, but those differences resolved by 12 and 24 months post-ORYGB, respectively.

Cardiopulmonary comorbidities by age in ORYGB are tabulated in Table 3. Baseline CHF, HTN, angina, LEE, PVD, dyslipidemia, OHS, and IHD varied directly with increasing age $(\mathrm{p}<0.01)$. DVT/
PE varied non-linearly with age. Twelve months post-ORYGB CHF, HTN, angina, PVD, and dyslipidemia varied directly with age $(\mathrm{p}<0.01)$; CHF, HTN, angina, and dyslipidemia $(\mathrm{p}<0.05)$ varied directly at 24 months. LEE and OSA varied non-linearly with age at 12 and 24 months post-operatively $(\mathrm{p}<0.05)$. PHTN increased directly with age only at 24 months. 
Table 2 Weight $(\mathrm{kg})$ and BMI +/- Std dev by age in ORYGB patients

\begin{tabular}{|c|c|c|c|c|c|c|c|c|}
\hline & $\begin{array}{l}\text { Age Group } \\
\text { (n) }\end{array}$ & $<30(591)$ & $30-40(1252)$ & $40-50$ (1527) & $50-60(1388)$ & $60-70(592)$ & $>70$ (39) & p-Value \\
\hline Preop & Weight (Kg) & $\begin{array}{l}147.42+/- \\
35.93\end{array}$ & $\begin{array}{l}\mid 45.58+/- \\
37.26\end{array}$ & $\begin{array}{l}\mid 42.23+/- \\
34.63\end{array}$ & $\begin{array}{l}|37.9|+/- \\
30.64\end{array}$ & $\begin{array}{l}130.43+/- \\
25.58\end{array}$ & $\begin{array}{l}123.43+/- \\
23.48\end{array}$ & $<.0001$ \\
\hline \multicolumn{9}{|c|}{ Comorbidities } \\
\hline & BMI & $\begin{array}{l}51.89+/- \\
10.76\end{array}$ & $\begin{array}{l}51.49+/- \\
10.86\end{array}$ & $\begin{array}{l}50.38+/- \\
10.10\end{array}$ & $49.48+/-9.09$ & $\begin{array}{l}47.47+/- \\
7.92\end{array}$ & $\begin{array}{l}45.40+/- \\
5.96\end{array}$ & $<.0001$ \\
\hline & Age Group (n) & $<30(164)$ & $30-40(4 \mid 9)$ & $40-50(566)$ & $50-60(616)$ & $60-70(305)$ & $>70(22)$ & $\mathrm{p}$-Value \\
\hline I2-Months & Weight (Kg) & $\begin{array}{l}92.97+/- \\
23.98\end{array}$ & $\begin{array}{l}93.49+/- \\
24.58\end{array}$ & $\begin{array}{l}93.69+/- \\
24.03\end{array}$ & $\begin{array}{l}92.12+/- \\
22.98\end{array}$ & $\begin{array}{l}87.84+/- \\
18.46\end{array}$ & $\begin{array}{l}86.17+/- \\
17.4\end{array}$ & $<.0001$ \\
\hline \multicolumn{9}{|l|}{ Post-ORYGB } \\
\hline & BMI & $\begin{array}{l}32.95+/- \\
7.74\end{array}$ & $33.19+/-7.54$ & $33.26+/-7.39$ & $32.91+/-6.95$ & $\begin{array}{l}32.27+/- \\
6.04\end{array}$ & $\begin{array}{l}32.17+/- \\
4.57\end{array}$ & $<.0001$ \\
\hline & Age Group (n) & $<30(47)$ & $30-40(111)$ & $40-50(189)$ & $50-60(205)$ & $60-70(129)$ & $>70(\mathrm{II})$ & $\mathrm{p}$-Value \\
\hline 24-Months & Weight (Kg) & $\begin{array}{l}87.94+/- \\
20.64\end{array}$ & $\begin{array}{l}87.38+/- \\
21.21\end{array}$ & $88.77+/-23.8$ & $90.9+/-24.89$ & $\begin{array}{l}84.82+/- \\
17.38\end{array}$ & $\begin{array}{l}83.76+/- \\
13.11\end{array}$ & $<.0001$ \\
\hline Post-ORYGB & & & & & & & & \\
\hline
\end{tabular}

Table 3 Cardiopulmonary comorbidities (\%) by age in ORYGB patients

\begin{tabular}{|c|c|c|c|c|c|c|c|c|}
\hline & Age group (n) & $<30(591)$ & $30-40$ (I 252) & $40-50(1527)$ & $50-60(1388)$ & $60-70(592)$ & $>70$ (39) & p-Value \\
\hline & DVT/PE & 1.69 & 2.4 & 3.93 & 5.19 & 4.73 & 5.13 & 0.0003 \\
\hline Preop & $\mathrm{CHF}$ & 0.51 & 2.08 & 2.03 & 4.39 & 7.43 & 0 & $<.0001$ \\
\hline \multirow[t]{11}{*}{ Comorbidities } & HTN & 34.69 & 46.09 & 63 & 76.51 & 82.6 & 89.74 & $<.0001$ \\
\hline & ANGINA & 1.52 & 2.96 & 3.54 & 4.83 & 5.07 & 0 & 0.0014 \\
\hline & LEE & 12.01 & 21.49 & 28.03 & 33.36 & 33.61 & 15.38 & $<.0001$ \\
\hline & PVD & 1.02 & 0.48 & 1.05 & 2.31 & 3.55 & 5.13 & $<.0001$ \\
\hline & DYSLIPIDEMIA & $|5.9|$ & 25 & 39.69 & 53.31 & 58.28 & 53.85 & $<.0001$ \\
\hline & $\mathrm{OHS}$ & 2.03 & 2.88 & 2.82 & 3.89 & 5.41 & 5.13 & 0.011 \\
\hline & OSA & 36.04 & 43.77 & 55.53 & 54.76 & 52.36 & 38.46 & $<.0001$ \\
\hline & ASTHMA & 22.34 & 18.85 & 19.58 & 22.55 & 20.61 & 10.26 & 0.07 \\
\hline & PHTN & 2.2 & 4.07 & 3.8 & 5.04 & 4.73 & 5.13 & 0.0881 \\
\hline & IHD & 1.02 & 2.4 & 4.32 & 8.21 & 10.3 & 10.26 & $<.0001$ \\
\hline & Age Group (n) & $<30(164)$ & $30-40$ (419) & $40-50(566)$ & $50-60(616)$ & $60-70(305)$ & $>70(22)$ & $\mathrm{p}$-Value \\
\hline 12-Months & $\mathrm{CHF}$ & 0.61 & 1.19 & $1.4 \mathrm{I}$ & 4.22 & 7.21 & 0 & $<.0001$ \\
\hline \multirow[t]{10}{*}{ Post-ORYGB } & HTN & 25 & 27.68 & 36.4 & 53.41 & 64.59 & 68.18 & $<.0001$ \\
\hline & ANGINA & 0 & 2.86 & 1.24 & 3.41 & 4.26 & 4.55 & 0.0157 \\
\hline & LEE & 7.32 & 15.04 & 18.37 & 21.92 & 21.31 & 13.64 & 0.0002 \\
\hline & PVD & 0.61 & 0.72 & 1.24 & 1.79 & 4.26 & 9.09 & 0.0004 \\
\hline & DYSLIPIDEMIA & 14.63 & 17.18 & 22.97 & 37.99 & 44.92 & 59.09 & $<.0001$ \\
\hline & OHS & 1.83 & 1.43 & 0.53 & 1.95 & 2.3 & 0 & 0.2795 \\
\hline & OSA & 26.83 & 22.67 & 33.04 & 35.23 & 32.13 & 31.82 & 0.0007 \\
\hline & ASTHMA & 20.12 & 11.93 & 15.37 & 16.07 & 14.75 & 13.64 & 0.2184 \\
\hline & PHTN & 0 & 1.19 & 0.88 & 2.11 & 1.97 & 4.55 & 0.1626 \\
\hline & Age Group (n) & $<30(47)$ & $30-40$ (III) & $40-50$ (I89) & $50-60(205)$ & $60-70(129)$ & $>70(\mathrm{II})$ & $\mathrm{p}$-Value \\
\hline
\end{tabular}


Table Continued..

\begin{tabular}{|c|c|c|c|c|c|c|c|c|}
\hline & Age group (n) & $<30(591)$ & $30-40(1252)$ & $40-50(1527)$ & $50-60(1388)$ & $60-70(592)$ & $>70$ (39) & p-Value \\
\hline 24-Months & $\mathrm{CHF}$ & 0 & 0 & 1.59 & 3.9 & 6.98 & 9.09 & 0.0104 \\
\hline \multirow[t]{9}{*}{ Post-ORYGB } & HTN & 27.66 & 27.93 & 31.22 & 47.32 & 58.91 & 54.55 & $<.0001$ \\
\hline & ANGINA & 0 & 0 & 1.59 & 3.41 & 5.43 & 9.09 & 0.0438 \\
\hline & LEE & 10.64 & 5.41 & 12.17 & 20.49 & 17.83 & 0 & 0.003 \\
\hline & PVD & 0 & 0 & 1.59 & 0.98 & 4.65 & 0 & 0.0523 \\
\hline & DYSLIPIDEMIA & 17.02 & 15.32 & 21.16 & 28.29 & 40.31 & 45.45 & $<.0001$ \\
\hline & OHS & 2.13 & 0.9 & 0 & 0.98 & 0.78 & 0 & 0.7021 \\
\hline & OSA & 23.4 & $|3.5|$ & 24.34 & 31.22 & 27.13 & 9.09 & 0.0157 \\
\hline & ASTHMA & 17.02 & 7.21 & 15.87 & 13.66 & 21.71 & 18.18 & 0.0632 \\
\hline & PHTN & 0 & 0 & $\mid .31$ & 0.87 & 0 & 9.09 & 0.0135 \\
\hline
\end{tabular}

Endocrine, metabolic, and somatic comorbidities by age in ORYGB patients are tabulated in Table 4. Baseline, 12, and 24 month post-ORYGB PCOS and PTC vary inversely with age $(\mathrm{p}<0.01)$. Diabetes (baseline, 12, and 24 months post-operatively) and gout

Table 4 Endocrine, metabolic, \& somatic comorbidities (\%) by age in ORYGB patients

\begin{tabular}{|c|c|c|c|c|c|c|c|c|}
\hline & $\begin{array}{l}\text { Age Group } \\
\text { (n) }\end{array}$ & $<30(591)$ & $30-40$ ( 1252$)$ & $40-50$ (1527) & $50-60(1388)$ & $60-70(592)$ & $>70$ (39) & p-Value \\
\hline Preop & Diabetes & 14.04 & 26.28 & 40.8 & 52.23 & 53.72 & 61.54 & $<.0001$ \\
\hline \multirow[t]{8}{*}{ Comorbidities } & Gout & 1.18 & 2.24 & 3.93 & 5.48 & 5.41 & 7.69 & $<.0001$ \\
\hline & PCOS & 10.83 & 6.95 & 2.95 & 0.72 & 0.17 & 0 & $<.0001$ \\
\hline & PTC & 3.21 & 2.72 & 2.75 & $\mathrm{I} .08$ & 0.68 & 0 & 0.0004 \\
\hline & Back Pain & 47.38 & 51.28 & 49.77 & 52.38 & 52.2 & 41.03 & 0.2316 \\
\hline & Fibromyalgia & 0.51 & 2.72 & 4.45 & 4.47 & 4.22 & 2.56 & $<.0001$ \\
\hline & IFS & 1.52 & 3.19 & 5.11 & 8.57 & 10.64 & 5.13 & $<.0001$ \\
\hline & MSP & 27.92 & 33.31 & 41.26 & 47.26 & 44.93 & 28.21 & $<.0001$ \\
\hline & Age Group (n) & $<30(164)$ & $30-40$ (4I9) & $40-50(566)$ & $50-60(616)$ & $60-70(305)$ & $>70(22)$ & $\mathrm{p}$-Value \\
\hline I2-Months & Diabetes & 9.15 & 12.17 & 15.72 & 28.57 & 27.54 & 50 & $<.0001$ \\
\hline \multirow[t]{7}{*}{ Post-ORYGB } & Gout & 1.22 & 2.39 & 1.59 & 4.71 & 3.93 & 13.64 & 0.0008 \\
\hline & PCOS & 9.15 & 6.44 & 4.06 & 0.49 & 0.66 & 0 & $<.0001$ \\
\hline & PTC & 3.05 & 1.43 & $1.4 \mathrm{I}$ & 0.32 & 0.33 & 0 & 0.0288 \\
\hline & Back Pain & 39.02 & 36.52 & 37.46 & 45.45 & 42.62 & 45.45 & 0.0329 \\
\hline & IFS & 0.61 & 2.39 & 3.89 & 7.47 & 9.84 & 0 & $<.0001$ \\
\hline & MSP & 21.34 & 20.53 & 28.45 & 39.77 & 35.41 & 36.36 & $<.0001$ \\
\hline & Age Group (n) & $<30(47)$ & $30-40(\mathrm{III})$ & $40-50$ (189) & $50-60(205)$ & $60-70$ (129) & $>70(\mathrm{II})$ & $\mathrm{p}$-Value \\
\hline 24-Months & Diabetes & 8.51 & 9.91 & 13.23 & $19.5 \mid$ & 24.03 & 18.18 & 0.0161 \\
\hline \multirow[t]{5}{*}{ Post-ORYGB } & Gout & 4.26 & 1.8 & 2.65 & 3.9 & 3.88 & 18.18 & 0.1224 \\
\hline & PTC & 4.26 & 2.7 & 2.12 & 0.49 & 0 & 0 & 0.1671 \\
\hline & Back Pain & 46.81 & 36.04 & 38.1 & 44.39 & 44.19 & 36.36 & 0.5468 \\
\hline & IFS & 4.26 & 0 & 3.7 & 9.76 & 10.08 & 18.18 & 0.0014 \\
\hline & MSP & 14.89 & 18.02 & 25.93 & 37.56 & 31.01 & 45.45 & 0.0008 \\
\hline
\end{tabular}

Citation: Menon A, Kelter D, Slotman GJ. Pre-operative direct and inverse clinical characteristic variations by age do not resolve up to 24 months following open roux-en-y gastric bypass. Adv Obes Weight Manag Control. 202 I;I I (5):I46-I 53. DOI: I0.15406/aowmc.202 I.I I.00349

(baseline and 12 months post-operatively) varied directly with age $(\mathrm{p}<0.01)$. Baseline, 12 , and 24 months post-ORYGB MSP varied nonlinearly as did 12 months back pain. 
Abdominal and hepatobiliary comorbidities by age in ORYGB patients are presented in Table 5. Baseline hernia, cholelithiasis, GERD, panniculitis, liver disease, and SUI varied non-linearly with age. At 12 months cholelithiasis and SUI varied directly with age. Abdominal hernia varied indirectly at 24 months.

Table 5 Abdominal \& Hepatobiliary Comorbidities (\%) by Age in ORYGB Patients

\begin{tabular}{|c|c|c|c|c|c|c|c|c|}
\hline & Age Group (n) & $<30(591)$ & $30-40(1252)$ & $40-50(1527)$ & $50-60(1388)$ & $60-70(592)$ & $>70$ (39) & p-Value \\
\hline Preop & Abd Hernia & 1.52 & 5.03 & 8.71 & 10.59 & 9.97 & 5.13 & $<.0001$ \\
\hline \multirow[t]{5}{*}{ Comorbidities } & Cholelithiasis & 9.48 & 14.7 & 19.91 & 25.36 & 23.82 & 17.95 & 0.0183 \\
\hline & GERD & 36.72 & 38.66 & 42.96 & 47.12 & 42.4 & 43.59 & $<.0001$ \\
\hline & Abd Panniculitis & 6.09 & 7.43 & 8.19 & 10.01 & 6.25 & 7.69 & $<.0001$ \\
\hline & SUI & $12.0 \mid$ & 16.85 & 20.63 & 24.14 & 25 & 17.95 & $<.0001$ \\
\hline & Age Group (n) & $<30(164)$ & $30-40(419)$ & $40-50(566)$ & $50-60(616)$ & $60-70(305)$ & $>70(22)$ & $\mathrm{p}$-Value \\
\hline I2-Months & Abd Hernia & 1.22 & 6.44 & 9.19 & 9.25 & 9.18 & 4.55 & 0.0104 \\
\hline \multirow[t]{6}{*}{ Post-ORYGB } & Cholelithiasis & 14.63 & 14.32 & 18.73 & 22.73 & 22.3 & 31.82 & 0.0034 \\
\hline & GERD & 23.78 & 21.72 & 26.33 & 30.03 & 24.92 & 18.18 & 0.0615 \\
\hline & Abd Panniculitis & 11.59 & 10.02 & 9.19 & 9.9 & 6.56 & 9.09 & 0.5167 \\
\hline & Liver Disease & 8.54 & 12.17 & 11.13 & 12.01 & 12.79 & 9.09 & 0.7901 \\
\hline & SUI & 8.54 & 10.02 & 13.07 & 14.94 & 18.69 & 13.64 & 0.0068 \\
\hline & Age Group (n) & $<30(47)$ & $30-40(1 \mathrm{I})$ & $40-50$ (189) & $50-60(205)$ & $60-70$ (129) & $>70(\mathrm{II})$ & $p$-Value \\
\hline 24-Months & Abd Hernia & 0 & 8.11 & 10.58 & 14.63 & 6.2 & 27.27 & 0.0064 \\
\hline \multirow[t]{5}{*}{ Post-ORYGB } & Cholelithiasis & 19.15 & 9.91 & 19.05 & 23.41 & 20.93 & 18.18 & 0.1184 \\
\hline & GERD & 27.66 & 24.32 & 29.1 & 33.17 & 26.36 & 9.09 & 0.3578 \\
\hline & Abd Panniculitis & 8.51 & 8.11 & 10.05 & 14.15 & 8.53 & 9.09 & 0.4999 \\
\hline & Liver Disease & 4.26 & 10.81 & 11.11 & 13.17 & 8.53 & 9.09 & 0.5413 \\
\hline & SUI & 14.89 & 7.21 & 12.7 & 12.2 & 20.16 & 18.18 & 0.0927 \\
\hline
\end{tabular}

Behavioral and psychiatric comorbidities by age are listed in Table 6. Alcohol and tobacco abuse, depression, PI, and MHD varied non-linearly by age at baseline. Unemployment varied directly. Post-
ORYGB depression varied non-linearly at 12 months and tobacco abuse varied non-linearly at both 12 and 24 months.

Table 6 Behavioral \& psychiatric comorbidities (\%) by Age in ORYGB patients

\begin{tabular}{|c|c|c|c|c|c|c|c|c|}
\hline & Age group (n) & $<30(591)$ & $30-40$ ( 1252$)$ & $40-50$ ( 1527$)$ & $50-60(1388)$ & $60-70(592)$ & $>70$ (39) & p-Value \\
\hline Preop & Alcohol abuse & & & & & & & 0.034 \\
\hline \multicolumn{9}{|c|}{ Comorbidities } \\
\hline & & 13.03 & 15.26 & 16.7 & 17.72 & 13.85 & 7.69 & \\
\hline & Tobacco abuse & 6.43 & 6.47 & 7.07 & 4.25 & 2.2 & 0 & $<.0001$ \\
\hline & Substance abuse & 0.68 & 0.24 & 0.13 & 0.43 & 0 & 0 & 0.1804 \\
\hline & Depression & 26.06 & 31.87 & 34.71 & 38.62 & $30.4 I$ & 7.69 & $<.0001$ \\
\hline & PI & 15.4 & $17.4 \mid$ & 19.97 & 18.88 & 14.19 & 2.56 & 0.0013 \\
\hline & MHD & 10.49 & 12.3 & 13.1 & 11.31 & 6.42 & 0 & 0.0001 \\
\hline & Unemployment & 17.77 & 19.09 & 25.67 & 32.35 & 51.69 & 51.28 & $<.0001$ \\
\hline & Age Group (n) & $<30(164)$ & $30-40$ (419) & $40-50(566)$ & $50-60(616)$ & $60-70(305)$ & $>70(22)$ & p-Value \\
\hline 12 Months & Alcohol abuse & & & & & & & 0.8922 \\
\hline
\end{tabular}

Citation: Menon A, Kelter D, Slotman GJ. Pre-operative direct and inverse clinical characteristic variations by age do not resolve up to 24 months following open roux-en-y gastric bypass. Adv Obes Weight Manag Control. 202 I;I I (5):I46-I 53. DOI: I0.15406/aowmc.2021.II.00349 
Table Continued...

\begin{tabular}{|c|c|c|c|c|c|c|c|c|}
\hline & Age group (n) & $<30(591)$ & $30-40(1252)$ & $40-50(1527)$ & $50-60(1388)$ & $60-70(592)$ & $>70$ (39) & p-Value \\
\hline \multicolumn{9}{|c|}{ Post-ORYGB } \\
\hline & & 10.37 & 12.89 & 11.84 & $|3.3|$ & 11.8 & 9.09 & \\
\hline & Tobacco abuse & 4.88 & 4.53 & 6.18 & 3.57 & 1.64 & 0 & 0.032 \\
\hline & Substance abuse & 1.22 & 0.48 & 0.18 & 0.32 & 0 & 0 & 0.3439 \\
\hline & Depression & 23.78 & 26.73 & 32.86 & 34.58 & 28.52 & 4.55 & 0.0012 \\
\hline & $\mathrm{PI}$ & 12.8 & 14.8 & 18.2 & 15.42 & 12.79 & 4.55 & 0.1578 \\
\hline & MHD & 9.15 & 10.02 & 11.48 & 9.74 & 4.59 & 0 & 0.0171 \\
\hline & Support Group & 15.24 & 18.1 & |7.11 & 15.26 & 11.48 & 9.09 & 0.1785 \\
\hline & Age Group (n) & $<30(47)$ & $30-40(111)$ & $40-50(189)$ & $50-60(205)$ & $60-70(129)$ & $>70(\mathrm{II})$ & $\mathrm{p}$-Value \\
\hline 24 Months & Alcohol abuse & & & & & & & 0.6322 \\
\hline \multicolumn{9}{|c|}{ Post-ORYGB } \\
\hline & & 12.77 & 7.21 & 12.17 & 13.66 & 10.08 & 9.09 & \\
\hline & Tobacco abuse & 12.77 & 6.31 & 6.35 & 3.41 & 0.78 & 9.09 & 0.0182 \\
\hline & Substance Abuse & 0 & 0.9 & 1.06 & 0.49 & 0.78 & 0 & 0.968 \\
\hline & Depression & 25.53 & 23.42 & 30.69 & 33.66 & 32.56 & 9.09 & 0.2387 \\
\hline & $\mathrm{PI}$ & 14.89 & 9.91 & 16.4 & 19.02 & 16.28 & 9.09 & 0.4229 \\
\hline & MHD & 10.64 & 5.41 & 10.05 & 11.22 & 12.4 & 0 & 0.4127 \\
\hline & Support Group & 19.15 & $|4.4|$ & 17.46 & 14.63 & 13.18 & 9.09 & 0.8311 \\
\hline
\end{tabular}

\section{Discussion}

The results of this study identify among ORYGB patients statistically and clinically significant pre-operative and outcomes variations by decade of age in weight, BMI, sex, health insurance, race, and 33 weight-related medical problems. Baseline weight, BMI, female/male, African-American and Hispanic race, Medicaid, Private, and Self-Pay insurance varied inversely to increasing age $(\mathrm{p}<0.01)$. Baseline Caucasian, Asian, and Other races varied non-linearly. Preoperative CHF, HTN, angina, LEE, PVD, dyslipidemia, OHS, IHD, diabetes, gout, IFS, unemployment varied directly with age, $<30$ to $60-70 />70$ years, while PCOS and PTC varied inversely. DVT/PE, OSA, hernia, cholelithiasis, GERD, panniculitis, liver disease, SUI, MSP, fibromyalgia, alcohol abuse, tobacco abuse, depression, PI, and MHD varied non-linearly among age groups. Variations by age in clinical characteristics of ORYGB patients did not resolve after surgery as CHF, HTN, angina, PVD, dyslipidemia, cholelithiasis, SUI, diabetes, gout, and IFS still varied directly with increasing age at 12 months, while PCOS and PTC varied inversely and LEE, OSA, hernia, MSP, tobacco abuse, depression, and MHD varied non-linearly. At 24 months, CHF, HTN, angina, dyslipidemia, diabetes, MSP, and IFS still varied directly with age. PTC varied inversely and LEE, OSA, PHTN, hernia, PCOS, and tobacco abuse varied non-linearly. This advance knowledge of variation in clinical characteristics and outcomes by decades of age in ORYGB may stimulate increased index of suspicion for these medically and surgically fragile patients, possibly facilitating optimal presumptive management. Although the years each patient carried obesity prior to ORYGB was not captured in BOLD, the direct correlations of most medically important obesity comorbidities with increasing age pre-operatively and the persistence of direct variations in clinically significant cardiopulmonary, endocrine/metabolic, somatic, and abdominal/hepatobiliary comorbidities is up to two years post-ORYGB implicates increasing age as a co-morbid factor. These data suggest that the longer one has obesity, the more entrenched become weight-related medical problems and the less effective is weight loss surgery in resolving them. Thus, older patients appear to benefit the least from ORYGB. Our review of the literature indicates that the significant age-related dyscrasias observed here among patients undergoing ORYGB have not been reported previously and are significant findings of this study.

While outcomes by insurance carrier were not analyzed in the present study, the higher prevalence of both Medicare and many life-threatening obesity co-morbidities among the oldest patients indirectly confirms previous investigations which observed impaired outcomes for older Medicare subscribers. ${ }^{6}$ Self-pay and private insurance were higher in younger age groups, while older populations were mainly Medicare. Results here indicate that younger patients are less burdened with obesity comorbidities than are older patients, which may be reflected in superior results following ORYGB.

Lyon et al previously reported that that outcomes for women were superior to men following LRYGB, manifested in female pre-operative comorbidities resolving with significantly higher percentages at 12 months when compared to males. ${ }^{5}$ Although focused on LRYGB, their findings are pertinent to this study, in which female/male percentage was higher in the $<30$ age group, decreasing linearly with age through 60-70 years, suggesting a link between sex and the age at which ORYGB was selected. These variables merit consideration in patient selection and optimization strategies for ORYGB.

Most pre-ORYGB cardiopulmonary, endocrine/metabolic, and somatic comorbidities in this report varied directly with age from $<30$ years until the $60-70 />70$ age groups, including the most serious weight-related problems of CHF, HTN, angina, dyslipidemia, OSA, and PHTN. These data confirm and expand upon the work of Major et al who noted higher rates of hypertension, diabetes mellitus, 
dyslipidemia, and OSA among older patients in LRYGB and sleeve gastrectomy. ${ }^{11-13}$ Although decreasing sample sizes in the $60-70 />70$ groups after ORYGB must be acknowledged, these persistent age variations support previously reported higher co-morbidity remission rates in younger bariatric surgery patients. ${ }^{14}$ This linear relationship with age is a result of compounding effects of age-related physiological changes in addition to environmental factors that predispose older populations to medical illnesses. Bariatric surgery is also effective in managing and alleviating symptoms of both PCOS and PTC, ${ }^{15,16}$ which supports our findings as both variables varied indirectly with age following ORYGB.

Pre-operative ORYGB abdominal and hepatobiliary comorbidities including abdominal hernia, cholelithiasis, GERD, panniculitis, liver disease, and SUI varied non-linearly with age, with highest incidences among 40-60 year old patients. At 12 months abdominal hernia and SUI still varied non-linearly, as did hernia at 24 months. Why abdominal/ hepatobiliary obesity co-morbidities varied non-linearly is not clear from the data. Since baseline disparities in BMI resolved early after ORYGB, BMI variation cannot be implicated at 12 and 24 months. Subichin et al studied the prevalence hepatobiliary disease processes in the morbidly obese and discovered additional independent risk factors such as age and tobacco abuse. ${ }^{17,18}$ Interestingly, Kim et al identified independent associations between RYGB and alcohol use with the subsequent development of hepatobiliary complications. ${ }^{19}$ Since alcohol consumption was highest also in the 40-60 years patients in the present study, drinking may have influenced increased liver disease. This suggests these variables likely play a role effecting outcomes following bariatric surgeries to support the non-linear age variations in this study.

Cholelithiasis varied non-linearly with age pre-operatively, then developed a direct correlation with increasing age at 12 months. Bariatric surgery is a well-known risk factor for the development of gallstones due to baseline obesity and rapid weight loss. ${ }^{20}$ However, advanced age is a causative factor in the development of biliary disease following bariatric surgery, which may explain the conversion of cholelithiasis to directly varying with age after ORYGB ${ }^{20,21}$ These findings may provide objective support for selective prophylactic cholecystectomy at the time of bariatric surgery in older patients.

A unique aspect of our study is the identification of age-independent variables following ORYGB, which include OSA and behavioral/ psychiatric variables such as tobacco abuse, depression, and MHD. With regards to OSA a non-linear age variation was discovered at baseline, 12, and 24-months post-operatively. Interestingly, the current literature depicts a weak association in older patients between OSA and metabolic syndrome as compared to younger populations, therefore substantiating our discovered non-linear age variation. ${ }^{22}$

Interactions of psychological and behavioral obesity comorbidities with ORYGB are not clear from the literature. In the present investigation these parameters affected the 40-60 years age groups most significantly at pre-operative baseline with depression and MHD continuing to vary non-linearly at 12 months, and tobacco abuse at 12 and 24 months post-ORYGB. White and co-authors reported that depression improves up to 24 months post-gastric ypass, and they associated high depression scores with sub-optimal weight loss. ${ }^{23}$ Conversely, Gott et al reported minimal improvement of psychological and behavioral co-morbidities after the five most common bariatric operations. ${ }^{24}$ Lifetime disorders of mood result in poorer weight loss outcomes following bariatric surgery. ${ }^{25}$ In addition, these variables are often affected by other disease processes and social history (drug use) ${ }^{26}$ Unfortunately, none of these finds nor the data here explain the significant psychological/behavioral problem concentration in 40-60 years ORGB patients Nevertheless, identifying and optimizing these variables pre-operatively may facilitate optimal post-ORYGB outcomes.

We present the first study analyzing a wide range of cardiovascular, abdominal, endocrine, metabolic, somatic, and behavioral/psychiatric by decade of age pre-operatively and in outcomes following ORYGB. Based on our data most of the weight-related medical problems following ORYGB are age dependent. From these results, one must consider the possibility that pursuing ORYGB in older populations may not be as advantageous as previously thought.

There were several limitations to our study. This is a retrospective analysis of prospectively collected data, and, as such, carries many typical confounders. In addition, the patients in this study represent a self-selected population who chose ORYGB, had this operation recommended for them, or had LRYGB converted to ORYGB. Therefore, the findings might not be extrapolated to other operations or to patients with obesity overall. Diagnosis of co-morbidities in BOLD was based on a clinical diagnosis or was self-reported by patients, rather than derived from a pathological diagnosis. For example, liver biopsy was not required, so liver disease was diagnosed clinically only.

\section{Conclusion}

The results of this study identify statistically and clinically significant pre-operative variations by age, both directly, inversely, as well as non-linearly, in clinical characteristics of patients with obesity who undergo ORYGB. These findings may raise index of suspicion for peri-operative medical risks in performing both weight-loss surgery and non-bariatric surgery, possibly leading to optimized morbidity, mortality, and outcomes for these fragile individuals. Variations by patient age pre-operatively in the most serious obesity co-morbidities of diabetes, CHF, HTN, angina, dyslipidemia, OSA, and PHTN persisted up to 24 months post ORYGB., suggesting that preoperative age influences ORYGB outcomes significantly Considering the surgical complexity and medical instability of ORYGB patients, the important variations by age identified in this study may provide bariatric surgeons additional pre-surgical understanding of the weightrelated medical problems and expected outcomes. The relative risks associated with age may supplement clinical judgement in planning for ORYGB. This advance clinical knowledge of age variation may facilitate ORYGB management.

\section{Acknowledgments}

The authors acknowledge the assistance of Nicole Stouffer, M.S. in statistical analysis.

\section{Conflicts of interest}

We disclose no conflicts of interest in this study.

\section{Funding}

None.

\section{References}

1. Michael L Schwartz, Raymond L Drew, Marilyn Chazin-Caldie. Factors determining conversion from laparoscopic to open Roux-en-Y gastric bypass. Obes Surg. 2004;14(9):1193-1197.

2. Nir Barzilai, Derek M Huffman, Radhika H Muzumdar,et al. The critical role of metabolic pathways in aging. Diabetes. 2012;61(6):1315-1322. 
3. Earl S Ford, Wayne H Giles, William H Dietz. Prevalence of the metabolic syndrome among US adults: findings from the third National Health and Nutrition Examination Survey. JAMA. 2002;287(3):356-359.

4. Magdalena Jura, Leslie P Kozak. Obesity and related consequences to ageing. Age (Dordr). 2016;38(1):23.

5. Malinda Lyon, Chris Bashian, Casey Sheck, et al. Outcomes following laparoscopic Roux-en-Y gastric bypass (LRYGB) vary by sex: Analysis of 83,059 women and men with morbid obesity. Am J Surg. 2019;217(6):1019-1024.

6. Michael Davis, Janette Gomez, Casey Sheck, et al. Variation in Weight and Obesity Comorbidities After Open Roux-en-Y Gastric Bypass by Health Insurance: Medicaid vs Medicare vs Private vs Self-Pay in 4225 Bariatric Outcomes Longitudinal Database Patients. JAMA Surg. 2017;152(1):105-109.

7. Michael H Wood, Arthur M Carlin, Amir A Ghaferi, et al. Association of Race With Bariatric Surgery Outcomes. JAMA Surg. 2019;154(5):e190029.

8. Jacob B Hammond, Christopher J Webb 1, Venkata S K K Pulivarthi, et al., Is There an Upper Age Limit for Bariatric Surgery? Laparoscopic Gastric Bypass Outcomes in Septuagenarians. Obes Surg. 2020.

9. Wayne J English, Eric J DeMaria, Matthew M Hutter, et al. American Society for Metabolic and Bariatric Surgery 2018 estimate of metabolic and bariatric procedures performed in the United States. Surg Obes Relat Dis. 2020;16(4):457-463.

10. SAS/STAT(R). 9.22 User's Guide. The SAS Institute, Cary, NC. 2009.

11. Raquel Gonzalez-Heredia, Neil Patel, Lisa Sanchez-Johnsen, et al. Does Age Influence Bariatric Surgery Outcomes? Bariatr Surg Pract Patient Care. 2015;10(2):74-78.

12. Piotr Major, Michał Wysocki, Michał Janik, et al. Impact of age on postoperative outcomes in bariatric surgery. Acta Chir Belg, 2018;118(5):307-314.

13. Emilie Montastier, Guillaume Becouarn, Emilie Bérard, et al. Gastric Bypass in Older Patients: Complications, Weight Loss, and Resolution of Comorbidities at 2 Years in a Matched Controlled Study. Obes Surg. 2016;26(8):1806-13.

14. M I Cooiman, E O Aarts, I M C Janssen, et al. Weight Loss, Remission of Comorbidities, and Quality of Life After Bariatric Surgery in Young Adult Patients. Obes Surg. 2019;29(6):1851-1857.

15. Ying-Jie Li, Yi Han, Bing He. Effects of bariatric surgery on obese polycystic ovary syndrome: a systematic review and meta-analysis. Surg Obes Relat Dis. 2019;15(6):942-950.
16. Joel D Handley, Bedanta P Baruah, David M Williams, et al., Bariatric surgery as a treatment for idiopathic intracranial hypertension: a systematic review. Surg Obes Relat Dis. 2015;11(6):1396-403.

17. Ala I Sharara, Luma Basma O Rustom, Halim Bou Daher, et al. Prevalence of gastroesophageal reflux and risk factors for erosive esophagitis in obese patients considered for bariatric surgery. Dig Liver Dis. 2019;51(10):1375-1379.

18. Michael Subichin, Jesse Clanton, Marta Makuszewski, et al. Liver disease in the morbidly obese: a review of 1000 consecutive patients undergoing weight loss surgery. Surg Obes Relat Dis. 2015;11(1):137-141.

19. Hannah P Kim, Yue Jiang, Timothy M Farrell, et al. Roux-en-Y Gastric Bypass Is Associated With Increased Hazard for De Novo Alcohol-related Complications and Liver Disease. J Clin Gastroenterol. 2021.

20. Julietta Chang, Ricard Corcelles, Mena Boules, et al. Predictive factors of biliary complications after bariatric surgery. Surg Obes Relat Dis. 2016;12(9):1706-1710.

21. Michelle R. Lent1, Peter N. Benotti, Tooraj Mirshahi, et al. All-Cause and Specific-Cause Mortality Risk After Roux-en-Y Gastric Bypass in Patients With and Without Diabetes. Diabetes Care. 2017;40(10):13791385 .

22. Jordan Gaines, Alexandros N Vgontzas, Julio Fernandez-Mendoza, et al. Obstructive sleep apnea and the metabolic syndrome: The road to clinically-meaningful phenotyping, improved prognosis, and personalized treatment. Sleep Med Rev. 2018; 42:211-219.

23. Marney A White, Melissa A Kalarchian, Michele D Levine, et al. Prognostic Significance of Depressive Symptoms on Weight Loss and Psychosocial Outcomes Following Gastric Bypass Surgery: A Prospective 24-Month Follow-Up Study. Obes Surg. 2015;25(10):p. 1909-16.

24. Melissa L. Gott, Paul R. Osterdahl, Casey Connors. Casey Connors, Gus Slotman, Bariatric Surgery Does Not Resolve Underlying Psychological/ Behavioral Problems in Patients with Morbid Obesity: Analysis of 166,601 Patients. Journal of the American College of Surgeons. 2019;229(4).

25. Melissa A Kalarchian, Marsha D Marcus, Michele D Levine, et al. Relationship of psychiatric disorders to 6-month outcomes after gastric bypass. Surg Obes Relat Dis. 2008;4(4):544-549.

26. Melissa A Kalarchian, Marsha D Marcus, Michele D Levine, et al Psychiatric disorders among bariatric surgery candidates: relationship to obesity and functional health status. Am J Psychiatry. 2007;164(2):328334. 